\title{
Strongly coupled bio-plasmonic system: Application to oxygen sensing
}

\author{
Shourya Dutta-Gupta and Olivier J. F. Martin ${ }^{\text {a) }}$ \\ Nanophotonics and Metrology Laboratory (NAM), EPFL, Lausanne (CH - 1015), Switzerland
}

(Received 8 March 2011; accepted 18 July 2011; published online 26 August 2011)

\begin{abstract}
We investigate theoretically the strong coupling between surface plasmon resonances (SPRs) and absorption bands of hemoglobin. When the surface plasmon resonance spectrally overlaps the absorption bands of hemoglobin, the system is strongly coupled and its dispersion diagram exhibits an anti-crossing. Working in the conditions of strong coupling enhances the sensitivity of a SPR sensor up to a factor of 10. A model for the permittivity of hemoglobin, both in oxygenated and deoxygenated states, is presented and the study is carried out for both angle and wavelength modulated SPR sensors. Finally, a differential measurement is shown to increase the sensitivity further. (C) 2011 American Institute of Physics. [doi:10.1063/1.3626786]
\end{abstract}

\section{INTRODUCTION}

Biosensors based on surface plasmon resonance (SPR) have been investigated extensively over the past few decades. ${ }^{1-6}$ It is now well understood that the excitation of SPRs leads to a large local field enhancement, ${ }^{3,7}$ which can facilitate the sensing, ${ }^{1,2,4,5}$ nonlinear optical, ${ }^{8,9}$ and cavity QED applications. Thus a change in the refractive index due to the presence of a biological entity causes a shift of the resonance position. By measuring this shift, one has an indication about properties such as refractive index, concentration, etc. of the biological entity. Some of the most prominent applications of such SPR sensors can be found in detection of biomolecules, viruses, bacteria, toxins, pesticides etc., especially when the concentration of the analyte is very low, parts per billion level or lower. ${ }^{4}$ Most SPR sensors have real time sensing ability that has been widely utilized for studying adsorption kinetics of molecules to a surface, ${ }^{2,5}$ proteinmembrane interactions, ${ }^{10}$ and live single cell imaging. ${ }^{11}$ Boussad et al. showed that it is also possible to detect changes in the conformation and the electronic state of redox proteins through multiwavelength SPR spectroscopy that is beyond the capabilities of optical absorption spectroscopy. ${ }^{12}$

Usually the SPR sensing does not require the antibody layer on the metal film to have optical resonances at the wavelength of the SPR. However, past studies have reported an enhancement in the sensitivity, if the antibody coating layer used for the detection of a specific antigen has an absorption band in the vicinity of the SPR resonance. ${ }^{13-17}$ Moreover, Nakkach et al. showed that the reflectivity variation can be enhanced by a factor of almost $160 \%$ by using appropriate dye molecules conjugated to the analyte (in this case DNA). ${ }^{17}$ It is worth mentioning that in these studies strong interaction or coupling between the absorption bands of the antibody layer and the surface plasmon was not observed. ${ }^{13,15-17}$ On the other hand, several studies have dealt with strong coupling between surface plasmons and strongly absorbing dye molecule but these studies have not

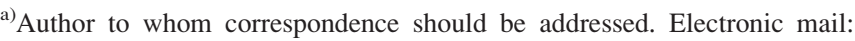
olivier.martin@epfl.ch.
}

illustrated how the strong coupling phenomenon is favorable for sensing applications. ${ }^{18-23}$

The phenomenon of strong coupling, which is typically characterized by an anti-crossing or avoided boundary crossing, is very well known and has been observed in different physical systems like cavity QED, ${ }^{24-29}$ plasmonics, and photonics $^{30-33}$ and has been applied for the development of practical applications like plasmonic switches ${ }^{34}$ and optical rulers. ${ }^{35}$ From the best of our knowledge, no studies have shown strong coupling between surface plasmons and common metallo-proteins (which generally have weak absorption bands as compared to dye molecules) like hemoglobin, myoglobin, etc. In this paper we show theoretically that it is possible to achieve strong coupling between surface plasmon and the weak absorption bands of hemoglobin ( $\mathrm{Hb}$ ). Moreover, we demonstrate how the presence of strong coupling between hemoglobin and surface plasmon can enhance the sensitivity of a standard SPR sensor for both wavelength and angle modulation modes of operation. It should be noted that oxygen detection using standard SPR (in which the metal is coated with a monolayer of the antibody) is extremely difficult since the oxygen induced refractive index change is negligibly small and thus the shift of the SPR is smaller than the detection limit of most instruments. Note that except for a few studies, where fluorescence enhancement from metallic surface was used, oxygen sensing has remained a challenging problem. ${ }^{36}$ In this paper we address this issue by proposing a method of detection for oxygen exploiting the enhanced sensitivity of a SPR sensor provided by strong coupling between the surface plasmon and $\mathrm{Hb}$ absorption bands.

The structure of the paper is as follows. In Sec. II we describe the system under study and recall the transfer matrix formalism for the analysis of such a layered structure. In the following section, we present the simulation results and demonstrate how the strong coupling can lead to enhanced sensitivity. Lastly, we summarize the main results of our investigation in Sec. IV.

\section{FORMULATION}

Consider the system shown in Fig. 1. The layered structure consists of a $50 \mathrm{~nm}$ silver (Ag) film and a $100 \mathrm{~nm}$ 


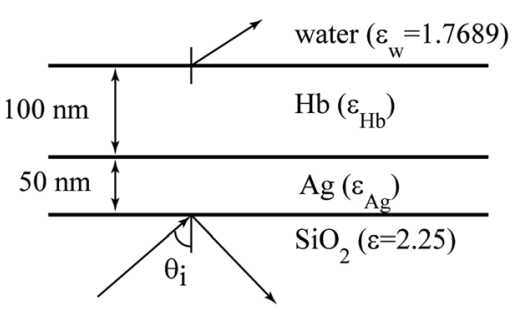

FIG. 1. Geometry of the system.

Hemoglobin $(\mathrm{Hb})$ layer stacked on top of a $\mathrm{SiO}_{2}$ slab $(\varepsilon=2.25)$. The dielectric function of silver is modeled using the data given by Johnson and Christy. ${ }^{37}$ The total concentration of $\mathrm{Hb}$ molecules in the Hb layer is kept fixed at $25 \mathrm{mM}$ for all the simulations ( $\mathrm{Hb}$ concentration in whole blood varies between 8 and $15 \mathrm{mM}$ ). We introduce a parameter $f_{r}$ $\left(0 \leq f_{r} \leq 1\right)$ that gives the fraction of $\mathrm{Hb}$ molecules that are in the oxygenated state, i.e., the $\mathrm{Hb}$ molecules that have $\mathrm{O}_{2}$ bound to them. Therefore,

$$
f_{r}=\frac{n_{\text {oxy }}}{n_{\text {oxy }}+n_{\text {deoxy }}},
$$

where $\mathrm{n}_{\text {oxy }}$ and $\mathrm{n}_{\text {deoxy }}$ are the number of oxygenated and deoxygenated $\mathrm{Hb}$ molecules in the film, respectively. For example, $\mathrm{f}_{\mathrm{r}}=1$ implies a completely oxygenated $\mathrm{Hb}$ layer whereas $f_{r}=0$ implies a completely deoxygenated Hb layer. The dielectric function of $\mathrm{Hb}$ (for completely oxygenated or deoxygenated $\mathrm{Hb}$ ) is modeled using the following empirical relation assuming a superposition of Lorentzian responses at three distinct frequencies

$$
\begin{aligned}
\varepsilon_{\alpha}= & \varepsilon_{w}+\frac{\nu_{p 1}^{2}}{\nu_{01}^{2}-\nu^{2}-i \gamma_{01} \nu}+\frac{\nu_{p 2}^{2}}{\nu_{02}^{2}-\nu^{2}-i \gamma_{02} \nu} \\
& +\frac{\nu_{p 3}^{2}}{\nu_{03}^{2}-\nu^{2}-i \gamma_{03} \nu}
\end{aligned}
$$

where $\alpha$ is oxy (oxygenated $\mathrm{Hb}$ ) or deoxy (deoxygenated $\mathrm{Hb}), \varepsilon_{w}$ is the dielectric constant of water $\left(\varepsilon_{w}=1.7689\right)$, and $\nu$ is the frequency of the incident light. The parameters $\nu_{0 \mathrm{~m}}$ $\left(=\mathrm{c} / \lambda_{0 \mathrm{~m}} ; c\right.$ is the speed of light in vacuum), $\nu_{p m}$ and $\gamma_{0 m}$ (where $m=1,2,3$ ) correspond to the position, strength, and damping of the various resonances, respectively. For the case when the $\mathrm{Hb}$ film is partly oxygenated $\left(0<f_{r}<1\right)$ we use a linear relation between $\varepsilon_{\text {oxy }}$ and $\varepsilon_{\text {deoxy }}$ to obtain the effective dielectric function of the $\mathrm{Hb}$ film:

$$
\varepsilon_{e f f}=f_{r} \varepsilon_{o x y}+\left(1-f_{r}\right) \varepsilon_{d e o x y} .
$$

The values of the parameters used in Eq. (2) are given in Table I for $\mathrm{Hb}$ concentration of $25 \mathrm{mM}$, they were obtained by comparing the absorption spectra obtained from Beer-

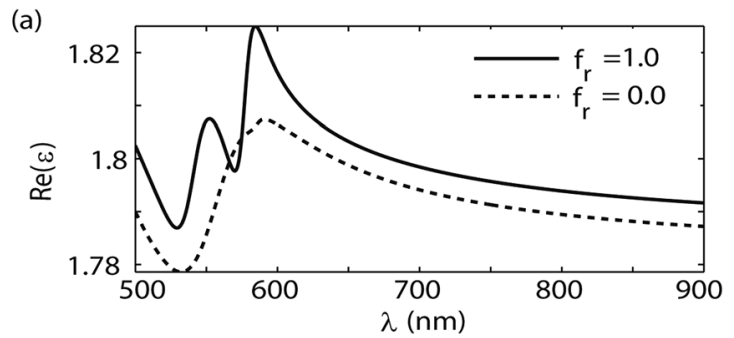

(b)

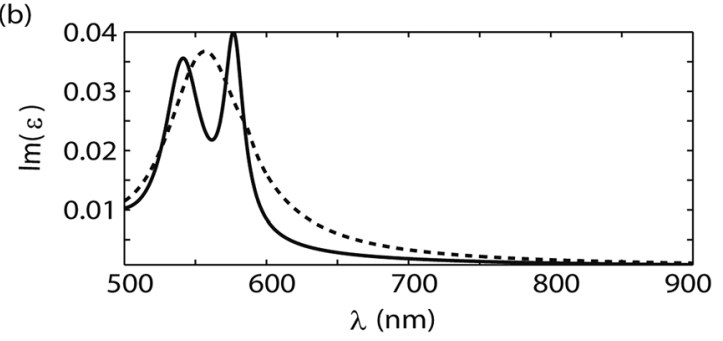

FIG. 2. (a) Real and (b) imaginary parts of the dielectric function of $\mathrm{Hb}$ at a concentration of $25 \mathrm{mM}$, in the oxygenated $\left(\mathrm{f}_{\mathrm{r}}=1\right)$ and deoxygenated $\left(f_{r}=0\right)$ states.

Lambert law (using the experimental data of the absorption coefficient $^{38}$ ) to the absorption spectra obtained using the transfer matrix method ${ }^{39,40}$ with normal incident light for a $100 \mathrm{~nm}$ film of $\mathrm{Hb}$ suspended in water, i.e., both the incident and the emergence medium is taken to be water.

The resonances at $\lambda_{01}$ and $\lambda_{02}\left(\lambda_{03}\right)$ correspond to the Q-bands (Soret band) of the absorption spectra of $\mathrm{Hb}$. Q-bands of the absorption spectra are of key interest in this paper because they exhibit a large change as a function of the oxygenation state of the Hb layer, i.e., change in $f_{r}$. It should be noted that in case of deoxygenated $\mathrm{Hb}$, the resonance at $\lambda_{02}$ is very weak as compared to the resonance at $\lambda_{01}$; whereas in case of oxygenated $\mathrm{Hb}$ the two resonances (at $\lambda_{01}$ and $\lambda_{02}$ ) are of comparable strength. Figure 2 shows the real and imaginary parts of the dielectric function of both oxygenated and deoxygenated $\mathrm{Hb}$ films. The effect of the absorption peaks on the effective dielectric function diminishes from the resonances toward higher wavelengths $\lambda$. Above $\lambda=800 \mathrm{~nm}$ the difference between the imaginary parts of $\varepsilon_{\text {oxy }}$ and $\varepsilon_{\text {deoxy }}$ is negligibly small $\left(\leq 5.36 \times 10^{-4}\right)$. In this regime i.e., when $\lambda \geq 800 \mathrm{~nm}$, there is a minimal contribution of the absorption bands of Hb toward the shift in the SPR peak caused by a change in $f_{r}$.

Let the structure in Fig. 1 be illuminated by a TM polarized plane wave (electric field in the plane of incidence) from the $\mathrm{SiO}_{2}$ side. In the Kretschmann-like configuration, the surface plasmon is generated at the $\mathrm{Ag}-\mathrm{Hb}$ interface. ${ }^{41}$

\begin{tabular}{|c|c|c|c|c|c|c|c|c|c|}
\hline & $\begin{array}{c}\nu_{p 1} \\
(\mathrm{THz})\end{array}$ & $\begin{array}{c}\nu_{p 2} \\
(\mathrm{THz})\end{array}$ & $\begin{array}{c}\nu_{p 3} \\
(\mathrm{THz})\end{array}$ & $\begin{array}{c}\gamma_{01} \\
(\mathrm{THz})\end{array}$ & $\begin{array}{c}\gamma_{02} \\
(\mathrm{THz})\end{array}$ & $\begin{array}{c}\gamma_{03} \\
(\mathrm{THz})\end{array}$ & $\begin{array}{c}\lambda_{01} \\
(\mathrm{~nm})\end{array}$ & $\begin{array}{c}\lambda_{02} \\
(\mathrm{~nm})\end{array}$ & $\begin{array}{c}\lambda_{03} \\
(\mathrm{~nm})\end{array}$ \\
\hline Oxygenated $\mathrm{Hb}$ & 23.5 & 15.8 & 87.0 & 32.5 & 15.0 & 39.0 & 541.0 & 577.0 & 415.0 \\
\hline Deoxygenated $\mathrm{Hb}$ & 35.5 & 3.0 & 64.5 & 66.0 & 10.0 & 20.0 & 556.0 & 586.0 & 434.0 \\
\hline
\end{tabular}
As the $\mathrm{Hb}$ layer experiences the SPR-induced large local field enhancement, one expects a strong coupling of the $\mathrm{Hb}$

TABLE I. Values of the various parameters used to fit the permittivity of $\mathrm{Hb}$. 
absorption bands with the SPR mode. This is further accentuated by the spectral proximity of these resonances.

The signature of this strong interaction can be seen in the reflection spectrum of the layered structure. To compute the reflection spectrum we use the transfer matrix formulation and recall briefly here the characteristic matrix results for the complex reflection $(r)$ and transmission coefficients $(t)$ for a layered structure ${ }^{39,40}$ :

$$
\begin{aligned}
r & =\frac{\left(m_{11}+m_{12} p_{f}\right) p_{i}-\left(m_{21}+m_{22} p_{f}\right)}{\left(m_{11}+m_{12} p_{f}\right) p_{i}+\left(m_{21}+m_{22} p_{f}\right)}, \\
t & =\frac{2 p_{i}}{\left(m_{11}+m_{12} p_{f}\right) p_{i}+\left(m_{21}+m_{22} p_{f}\right)},
\end{aligned}
$$

where $m_{q, r}(q, r=1,2)$ are the matrix elements of the total transfer matrix of the layered structure, which can be found in Ref. 39 and for TM polarization

$$
p_{k}=\sqrt{\mu_{k} / \varepsilon_{k}} \cos \theta_{k}, \quad k=i, f .
$$

Here $\theta_{k}$ are the angles of incidence $(k=i)$ and emergence $(k=f)$ and $\varepsilon_{k}$ and $\mu_{k}$ are the dielectric constant and relative permeability of the incident $(k=i)$ and the emergent media $(k=f)$, respectively. The reflectivity $(R)$ and transmissivity $(T)$ from the layered structure are given by

$$
\begin{aligned}
& R=|r|^{2}, \\
& T=\frac{p_{f}}{p_{i}}|t|^{2} .
\end{aligned}
$$

Since the total energy in the system is conserved, we have the following relation for absorption $(A)$ by the layered structure $A=1-R-T$.

One main advantage of using surface plasmons generated on thin metallic films is derived from their strong dispersion. As a consequence, the position of the resonance can be varied easily by changing the angle of incidence. This way one can spectrally overlap the SPR with the Hb absorption bands. Note that similar strong dispersive properties do not exist in the localized plasmons of metal nanoparticles or metal nanocomposites. The locations of the $\mathrm{Hb}$ resonances are more or less insensitive to the angle of incidence, while their comparative strengths can be controlled by concentration of $\mathrm{Hb}$ molecules in the $\mathrm{Hb}$ layer. On the other hand, the SPR location is very sensitive to the angle of incidence. The latter can be moved around by varying the angle of incidence resulting in strong coupling with the individual $\mathrm{Hb}$ modes for any value for $f_{r}$. In the next section, we present numerical results demonstrating this strong coupling.

\section{RESULTS AND DISCUSSIONS}

Figure 3(a) shows the dispersion relation (plot of $R$ versus $\theta$ and $\lambda$ ) of a surface plasmon excited on a thin metal film (geometry is the same as shown in Fig. 1, except that the Hb layer is absent). Figures 3(b) and 3(c) show the dispersion diagram of oxygenated and deoxygenated $\mathrm{Hb}$ layers, respectively (geometry is same as shown in Fig. 1 except that the Ag layer is absent). In this case we see that the absorption bands of $\mathrm{Hb}$ (both $f_{r}=0$ and $f_{r}=1$ ) are non-dispersive, i.e., exhibit no angular dependence of the resonance positions. Furthermore, the absorption band in case of deoxygenated $\mathrm{Hb}$ is broader, compared to that of oxygenated $\mathrm{Hb}$. By looking at the dispersion diagrams of the individual resonances, i.e., the surface plasmon resonance or the absorption bands of $\mathrm{Hb}$ (oxygenated or deoxygenated), it is clear that the individual systems do not possess an anti-crossing feature in their dispersion diagrams. Figures 3(d) and 3(e) shows the dispersion diagram of the complete system (Fig. 1) for the cases when $f_{r}=1$ and 0 , respectively. When $f_{r}=1$ the dispersion diagram exhibits two (a)

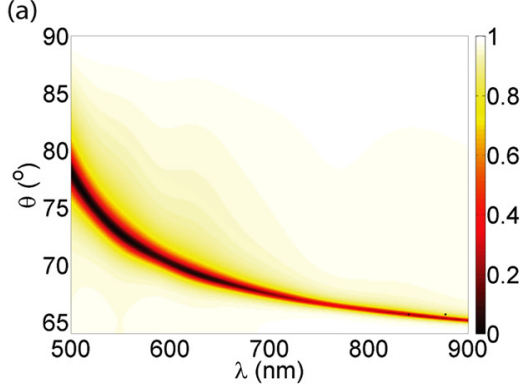

(b)

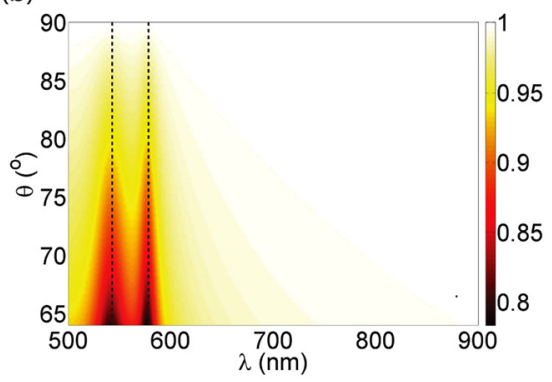

(c)

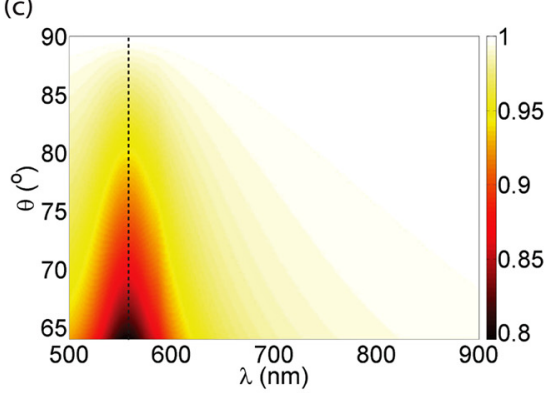

(d)

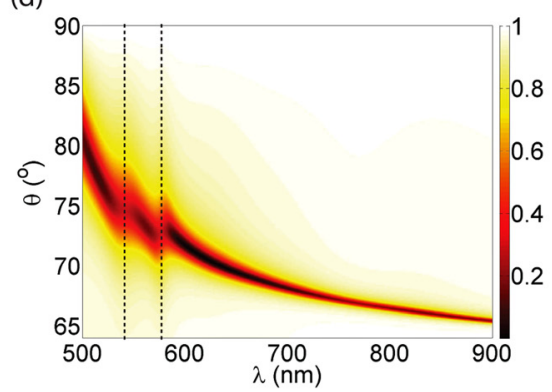

(e)

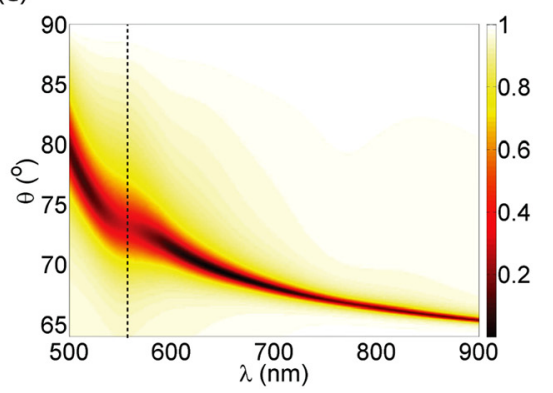

FIG. 3. (Color online) Dispersion diagram of (a) a thin silver film placed on a $\mathrm{SiO}_{2}$ substrate, (b) oxygenated $\mathrm{Hb}_{\text {layer }}$ placed on top of $\mathrm{SiO} \mathrm{O}_{2}$ slab, (c) deoxygenated $\mathrm{Hb}$ layer placed on top of $\mathrm{SiO}_{2}$ slab, (d) complete system (Fig. 1) when $\mathrm{f}_{\mathrm{r}}=1.0$, and (e) complete system (Fig. 1) when $\mathrm{f}_{\mathrm{r}}=0$. 
anti-crossings at the wavelengths of 541.0 and $577.0 \mathrm{~nm}$, which correspond to the absorption bands of uncoupled oxygenated $\mathrm{Hb}$. Similarly a single anti-crossing can be seen at around $\lambda=556.0 \mathrm{~nm}$ when $f_{r}=0$, which corresponds to the absorption band of deoxygenated $\mathrm{Hb}$. The presence of this kind of anti-crossings in the dispersion diagram is a clear indication of strong coupling between the surface plasmon and the absorption bands of oxygenated or deoxygenated $\mathrm{Hb}$. The resonances of the strongly coupled system are very different from those of the uncoupled system. For example, the absorption bands of oxygenated or deoxygenated $\mathrm{Hb}$ shift due to coupling. Another effect of coupling is that the absorption bands that are generally weak can be enhanced so that their detection becomes easier. The anti-crossings are more prominent in the case of oxygenated (Fig. 3(b)) compared to deoxygenated $\mathrm{Hb}$ (Fig. 3(c)) since the absorption bands in the former are sharper than in the latter. Another point of interest is that for a spectral region that is away from the anti-crossing region, i.e., for $\lambda>800.0 \mathrm{~nm}$, the two dispersion diagrams are very similar.

Now we demonstrate for both wavelength and angle modulated SPR sensors, ${ }^{42}$ that the shift of the resonance dips can be enhanced by working near the region of the strong coupling. Let us first consider a wavelength modulated SPR sensor, in which the angle of incidence is kept constant but the wavelength of incident light is changed so that reflectivity as a function of wavelength is measured. We compute the spectra at two different angles, $\theta_{i}=72.9^{\circ}$ and $66^{\circ}$. When the angle of incidence is $72.9^{\circ}$ the system is in the strong coupling regime, i.e., the surface plasmon mode and the absorption bands of $\mathrm{Hb}$ (oxygenated or deoxygenated) strongly couple, whereas when the angle of incidence is $66^{\circ}$ the system is decoupled. Figure 4(a) shows the reflectivity as a function of wavelength for the two chosen angles for both $f_{r}=0$ and 1 . From the figure it can be seen that three, respectively, two resonances are observed in the case of oxygenated, respectively, deoxygenated $\mathrm{Hb}$. We observe only two resonances in the case of deoxygenated layer even though it possesses three resonance dips (see Eq. (2)) since the resonance at $\lambda=586.0 \mathrm{~nm}$ is very weak and does not show up in the reflection spectra of the coupled system. When the system is strongly coupled, i.e., the angle of incidence is $72.9^{\circ}$, we see that when $f_{r}$ is changed from 1 to 0 the two resonances P1 (at $587.6 \mathrm{~nm}$ ) and P2 (at $564.8 \mathrm{~nm}$ ) converge to the resonance at $576.0 \mathrm{~nm}$, whereas the resonance P3 moves from $541.7 \mathrm{~nm}$ to $546.4 \mathrm{~nm}$. The position of the shoulder is given by the wavelength when $\partial^{2} R / \partial \lambda^{2}$ is maximum in the vicinity of the shoulder. Considering the decoupled case, i.e., when the incident angle is $66^{\circ}$, the resonance peak P4 shifts from $842.0 \mathrm{~nm}$ to $836.0 \mathrm{~nm}$ when $f_{r}$ changes from 1 to 0 . Figure 4(b) gives the relative shifts of the resonances as a function of the oxygenation of the Hb layer, i.e., $f_{r}$ varies from 0 to 1 . The shift is measured by taking $f_{r}=0$ as reference. From the figure it can be seen that the net shift (11.87 $\mathrm{nm}$ and $10.9 \mathrm{~nm}$ for $\mathrm{P} 1$ and $\mathrm{P} 2$ resonances) when the system is coupled is higher than when the system is decoupled (6.0 $\mathrm{nm}$ shift for P4 resonance). The peak P2 is followed from $f_{r}=0.1$ and not from $f_{r}=0$ because at very low values of $f_{r}$, distinguishing the resonances P1 and P2 becomes almost impossible. Also, rather than just looking at the shift of one resonance, we can look at the wavelength difference between two resonances, say P1 and P3, i.e.,

$$
\Delta \lambda=\left(\lambda_{P 1}-\lambda_{P 3}\right)-\left(\lambda_{P 1}\left(f_{r}=0\right)-\lambda_{P 3}\left(f_{r}=0\right)\right) .
$$

Such a differential measurement appears to be even more sensitive, as visible in Fig. 4(b), where the P1-P3 data shows the strongest wavelength shift as a function of $f_{r}$.

An alternate way of determining the change in $f_{r}$ can be by tracking the change in reflectivity of the resonance dips, as shown in Fig. 4(c). We can clearly see that if we measure the change in the reflectivity at the resonance P3 as a function of $f_{r}$, the change in reflectivity is much larger $(\Delta R=0.16)$ than in the decoupled case $(\Delta R=0.0244)$. Even when following the other two resonances, i.e., P1 and P2, for $f_{r}>0.3$ the change for these two resonances $(\Delta R=0.114$ and 0.068 for $\mathrm{P} 1$ and $\mathrm{P} 2$, respectively) is greater than that of the uncoupled case $(\Delta R=0.0244)$.

Let us now study the effect of strong coupling on the angular shifts for another commonly used mode of operation SPR sensors, namely angle modulation. In this mode of operation the incident wavelength is kept fixed (generally a laser source like He-Ne laser is used), whereas the angle of incidence is continuously varied. We considered for computation four different incident wavelengths: $541.0,577.0,556.0$, and $800.0 \mathrm{~nm}$. The first two wavelengths correspond to the absorption Q-bands of oxygenated $\mathrm{Hb}$, whereas the third wavelength corresponds to the absorption Q-band of deoxygenated $\mathrm{Hb}$. The last wavelength was chosen such that the system is not strongly coupled at this wavelength. Figures 5(a)-5(d) show the reflection spectra as a function of angle of incidence for the four different wavelengths. It is seen that the resonances are broader for wavelengths of $541.0 \mathrm{~nm}$ (Fig. 5 (a)), $556.0 \mathrm{~nm}$ (Fig. 5(b)), and $577.0 \mathrm{~nm}$ (Fig. 5(c)), compared to $800.0 \mathrm{~nm}$ (Fig. 5(d)). Figure 5(e) shows the angular shift of the resonance as a function of the oxygenation of the $\mathrm{Hb}$ film, i.e., when $f_{r}$ is varied from 0 to 1 . From the figure it is apparent (a)

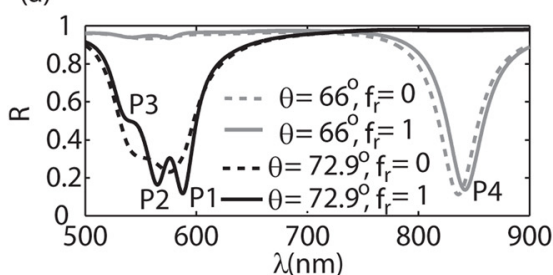

(b)

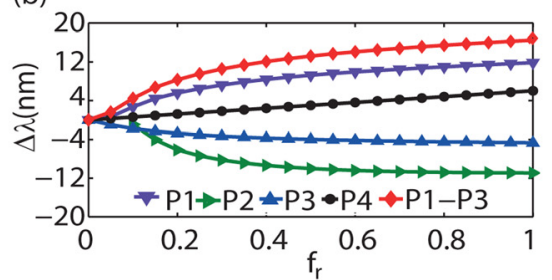

(c)

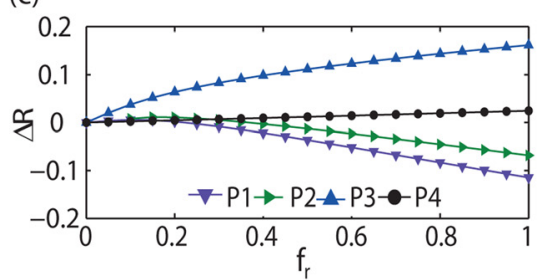

FIG. 4. (Color online) (a) Reflection spectra for two different angles of incidence; (b) shift of the resonance peak as a function of oxygenation for both angles of incidence; and (c) change in reflectivity as a function of the fraction of oxygenation for both angles of incidence. 
(a)

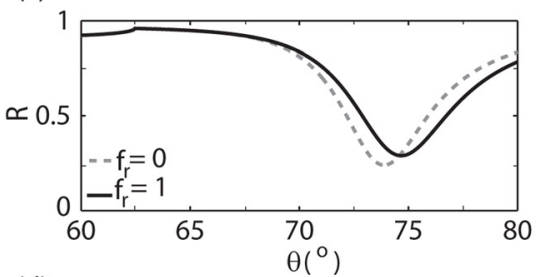

(d)

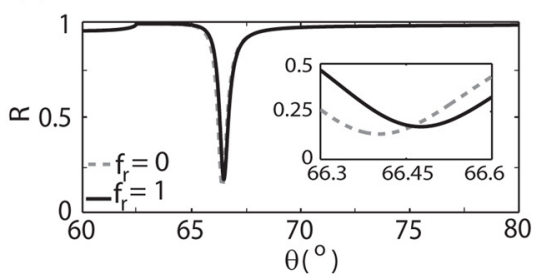

(b)

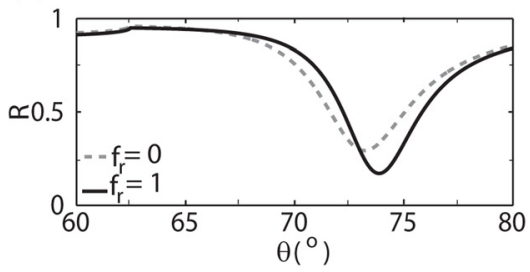

(e)

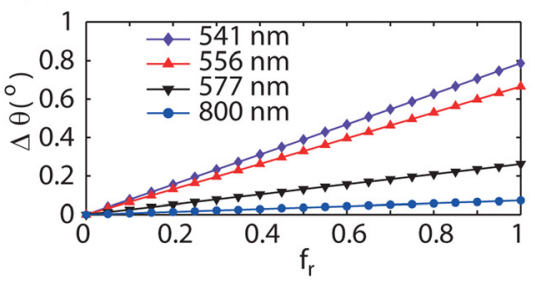

(c)

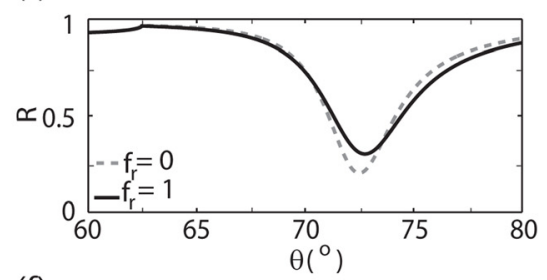

(f)

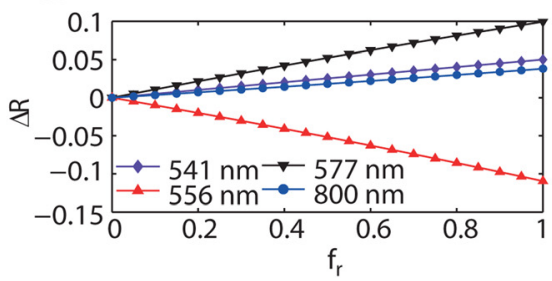

FIG. 5. (Color online) Reflectivity from the layered structure for four different wavelengths (a) $541 \mathrm{~nm}$, (b) $556 \mathrm{~nm}$, (c) $577 \mathrm{~nm}$, and (d) $800 \mathrm{~nm}$. (e) Angular shift as a function of oxygenation of the Hb layer for the four wavelengths; (f) change in reflectivity as a function of the fraction of oxygenation.

that when the wavelength is near the anti-crossing region, a larger angular shift $\left(\Delta \theta=0.787^{\circ}, 0.666^{\circ}\right.$, and $0.139^{\circ}$ for wavelengths of $541.0 \mathrm{~nm}, 556.0 \mathrm{~nm}$, and $577.0 \mathrm{~nm}$, respectively) is observed compared to the case when the chosen wavelength is away from the anti-crossing region (the shift is $0.073^{\circ}$ only for $\lambda=800 \mathrm{~nm}$ (inset of Fig. 5(d))). It can be seen that when strong coupling is present in the system, the angular shift is at least enhanced by a factor of two and, if the optimal incident wavelength is chosen, the enhancement factor can reach 10 times compared to the decoupled case. As in the case of wavelength modulation, we can also track the change of reflectivity at the resonance dip to obtain an estimate of $f_{r}$. This is shown in Fig. 5(f), which reports the reflectivity change of the resonance as a function of $f_{r}$ for the different incident wavelengths. It can be seen that the reflectivity change of the resonance is enhanced by the presence of strong coupling. In that case, the reflectivity change of the resonance dip is $0.050,0.110$, and 0.099 for incident wavelengths of $541.0 \mathrm{~nm}, 556.0 \mathrm{~nm}$, and $577.0 \mathrm{~nm}$, respectively; whereas for $\lambda=800.0 \mathrm{~nm}$ the change is only 0.038 when $f_{r}$ is varied from 0 to 1 .

By measuring the wavelength shift or the angular shift of the resonance dip, the change in the fraction $f_{r}$ or $\Delta f_{r}$ can be obtained. Thus the change in concentration of $\mathrm{Hb}$ molecules that has oxygen $\left(\mathrm{O}_{2}\right)$ bound to them can be inferred from the value of $\Delta f_{r}$. Let us carry out a brief discussion on the concentration of $\mathrm{O}_{2}$ that can be detected easily using both the coupled and the decoupled systems. If $\Delta f_{r}$ is being measured through wavelength shift or angular shift of the resonance dip, then the resolution of the instrument determines the lowest possible detectable concentration of $\mathrm{O}_{2}$. Since, 1 mole of $\mathrm{Hb}$ molecules can bind to four moles $\mathrm{O}_{2}$ molecules, the minimum resolvable concentration of $\mathrm{O}_{2}\left(\mathrm{C}_{\mathrm{O} 2, \mathrm{~min}}\right)$ is

$$
C_{O_{2}, \min }=4\left(\Delta f_{r}\right)_{\min } C
$$

where $\left(\Delta f_{r}\right)_{\min }$ is the minimum change in the fraction $f_{r}$ that can be resolved from the shift of the resonance dip and $C$ is the total concentration of $\mathrm{Hb}$ molecules $(C=25 \mathrm{mM})$. First consider a wavelength modulated SPR sensor with a wavelength resolution of $0.2 \mathrm{~nm}$. In this system for a wavelength shift of $0.2 \mathrm{~nm}$ to occur $f_{r}$ changes by 0.01 (following reso- nance P3, Fig. 4(b)) and 0.035 (following resonance P4, Fig. 4(b)) for the coupled and the decoupled case, respectively (note that the initial value of $f_{r}$ is 0 ). When the system is strongly coupled, i.e., the incident angle is $72.9^{\circ}$, then the value of $C_{O 2, \min }$ is $1.0 \mathrm{mM}$ whereas when the system is not strongly coupled, i.e., the incident angle is $66^{\circ}$, then the value of $C_{O 2 \text {, min }}$ is $3.5 \mathrm{mM}$. The minimum detectable amount in the presence of strong coupling is four times lower than the decoupled case. Now consider the case of an angle modulated SPR sensor having an angular resolution of $0.001^{\circ}$. A angular shift of $0.001^{\circ}$ is caused when $f_{r}$ changes by 0.00125 $(\lambda=541.0 \mathrm{~nm}$, Fig. 5(e) $)$ and $0.01400(\lambda=800.0 \mathrm{~nm}$, Fig. $5(\mathrm{e})$ ) for the coupled and the decoupled case, respectively (note that the initial $f_{r}$ value is 0 ). When the system is strongly coupled, i.e., the incident wavelength is $541.0 \mathrm{~nm}$, then the value of $C_{O 2, \min }$ is $0.125 \mathrm{mM}$ whereas when the system is not strongly coupled, i.e., the incident wavelength is $800 \mathrm{~nm}$, then the value of $C_{O 2, \min }$ is $1.4 \mathrm{mM}$. In this case the minimum detectable amount in the presence of strong coupling is almost 10 times smaller than in the decoupled case.

\section{CONCLUSIONS}

In conclusion, we have shown that strong coupling between a surface plasmon resonance and the absorption bands of metallo-proteins such as $\mathrm{Hb}$ is feasible. Furthermore, it has been demonstrated that this strong coupling can be exploited to enhance the sensitivity of a conventional SPR sensor. Numerical simulations based on transfer matrix approach show that for a wavelength modulated sensor the resonance shift is enhanced by a factor of 3 , whereas in the case of angle modulated sensor the resonance shift can be enhanced by a factor of 10 . Finally this sensitivity enhancement can be exploited for oxygen sensing.

\section{ACKNOWLEDGMENTS}

It is a pleasure to acknowledge stimulating discussions with G. Suarez and C. Santschi. This work was supported by the Swiss National Science Foundation (Grant No. CR23I2_130164). 
${ }^{1}$ J. Homola, S. S. Yee, and G. Gauglitz, Sens. Actuators B 54, 3 (1999). ${ }^{2}$ R. J. Green, R. A. Frazier, K. M. Shakesheff, M. C. Davies, C. J. Roberts, and S. J. B. Tendler, Biomater. 21, 1823 (2000).

${ }^{3}$ Surface Plasmon Resonance Based Sensors, Vol. 4, edited by J. Homola (Springer, New York, 2006).

${ }^{4}$ I. Abdulhalim, M. Zourob, and A. Lakhtakia, Electromagn. 28, 214 (2008).

${ }^{5}$ S. Scarano, M. Mascini, A. P. F. Turner, and M. Minunni, Biosens. Bioelect. 25, 957 (2010).

${ }^{6}$ M. Vala, K. Chadt, M. Piliarik, and J. Homola, Sens. Actuators B 148, 544 (2010).

${ }^{7}$ H. Raether, Surface Plasmons on Smooth and Rough Surfaces and on Gratings (Springer, New York, 1988).

${ }^{8}$ H. J. Simon, D. E. Mitchell, and J. G. Watson, Phys. Rev. Lett. 33, 1531 (1974).

${ }^{9}$ C.-C. Tzeng and J. T. Lue, Surf. Sci. 216, 579 (1989).

${ }^{10}$ M. Besenicar, P. Macek, J. H. Lakey, and G. Anderluh, Chem. Phys. Lipids 141, 169 (2006)

${ }^{11}$ Y. Yanase, T. Hiragun, S. Kaneko, H. J. Gould, M. W. Greaves, and M. Hide, Biosens, Bioelect, 26, 674 (2010).

${ }^{12}$ S. Boussaad, J. Pean, and N. J. Tao, Analyt. Chem, 72, 222 (2000).

${ }^{13}$ H. Kano and S. Kawata, Appl. Optics 33, 5166 (1994).

${ }^{14}$ A. Hanning, J. Roeraade, J. J. Delrow, and R. C. Jorgenson, Sens. Actuators B 54, 25 (1999)

${ }^{15}$ S. Wang, S. Boussaad, and N. J. Tao, Rev. Sci. Instrum. 72, 3055 (2001).

${ }^{16}$ K. Kurihara and K. Suzuki, Analyt. Chem. 74, 696 (2002).

${ }^{17}$ M. Nakkach, P. Lecaruyer, F. Bardin, J. Sakly, Z. B. Lakhdar, and M. Canva, Appl. Optics 47, 6177 (2008).

${ }^{18}$ J. Dintinger, S. Klein, F. Bustos, W. L. Barnes, and T. W. Ebbesen, Phys. Rev. B 71, 035424 (2005).

${ }^{19}$ T. Ambjörnsson, G. Mukhopadhyay, S. P. Apell, and M. Käll, Phys. Rev. B 73, 085412 (2006).

${ }^{20}$ C. Bonnand, J. Bellessa, and J.-C. Plénet, J. Non-Cryst. Solids 353, 1683 (2006).

${ }^{21}$ N. T. Fofang, T.-H. Park, O. Neumann, N. A. Mirin, P. Nordlander, and N. J. Halas, Nano Lett. 8, 3481 (2008).
${ }^{22}$ N. I. Cade, T. Ritman-Meer, and D. Richards, Phys. Rev. B 79, 241404 (2009).

${ }^{23}$ W. Ni, T. Ambjörnsson, S. P. Apell, H. Chen, and J. Wang, Nano Lett. 10, 77 (2010).

${ }^{24}$ J. J. Sanchez-Mondragon, N. B. Narozhny, and J. H. Eberly, Phys. Rev. Lett. 51, 550 (1983).

${ }^{25}$ G. S. Agarwal, J. Opt. Soc. Am. B 2, 480 (1985).

${ }^{26}$ M. G. Raizen, R. J. Thompson, R. J. Brecha, H. J. Kimble, and H. J. Carmichael, Phys. Rev. Lett. 63, 240 (1989).

${ }^{27}$ S. D. Gupta and G. S. Agarwal, Opt. Commun. 115, 597 (1995).

${ }^{28}$ D. G. Lidzey, D. D. C. Bradley, M. S. Skolnick, T. Virgili, S. Walker, and D. M. Whittaker, Nature 395, 53 (1998)

${ }^{29}$ J. R. Tischler, M. S. Bradley, Q. Zhang, T. Atay, A. Nurmikko, and V. Bulovic, Org. Elect. 8, 94 (2007).

${ }^{30}$ A. Christ, T. Zentgraf, S. G. Tikhodeev, N. A. Gippius, O. J. F. Martin, J. Kuhl, and H. Giessen, Physica Status Solidi B 243, 2344 (2006).

${ }^{31}$ A. Christ, Y. Ekinci, H. H. Solak, N. A. Gippius, S. G. Tikhodeev, and O. J. F. Martin, Phys. Rev. B 76, 201405 (2007)

${ }^{32}$ A. Christ, G. Lévéque, O. J. F. Martin, T. Zentgraf, J. Kuhl, C. Bauer, H. Giessen, and S. G. Tikhodeev, J. Microsc.229, 344 (2008).

${ }^{33}$ T. Schwartz, J. A. Hutchison, C. Genet, and T. W. Ebbesen, Phys. Rev. Lett. 106, 196405 (2011).

${ }^{34}$ T. Ming, L. Zhao, M. Xiao, and J. Wang, Small 6, 2514 (2010).

${ }^{35}$ C. Sönnichsen, B. M. Reinhard, J. Liphardt, and A. P. Alcisatos, Nat. Biotechnol. 23, 741 (2005).

${ }^{36}$ D. S. Smith, Y. Kostov, and G. Rao, Sens. Actuators B 127, 432 (2007).

${ }^{37}$ P. B. Johnson and R. W. Christy, Phys. Rev. B 6, 4370 (1972).

${ }^{38} \mathrm{~S}$. Prahl, see http://omlc.ogi.edu/spectra/hemoglobin/ (1999) for more information on the optical absorption of Hemoglobin.

${ }^{39} \mathrm{M}$. Born and E. Wolf, Principles of Optics: Electromagnetic Theory of Propagation (Cambridge University Press, Cambridge, 2001).

${ }^{40}$ S. D. Gupta, Progress in Optics (Nonlinear Optics of Stratified Media) (North Holland, Amsterdam, 1998), Vol. 38.

${ }^{41}$ E. Kretschmann and H. Raether, Z. Naturforsch. A 23, 2135 (1968).

${ }^{42}$ X. Liu, D. Song, Q. Zhang, Y. Tian, L. Ding, and H. Zhang, Trends Analyt. Chem.24, 887 (2005). 